(2) Open Access Full Text Article

\title{
Low body temperature associated with severe ischemic stroke within 6 hours of onset: The Bergen NORSTROKE Study
}

This article was published in the following Dove Press journal:

Vascular Health and Risk Management

3I May 2012

Number of times this article has been viewed

\section{Christopher E Kvistad \\ Lars Thomassen \\ Ulrike Waje-Andreassen \\ Halvor Naess \\ Department of Neurology, Haukeland University Hospital, University of Bergen, Bergen, Norway}

Background: Hypothermia is considered neuroprotective and a potential treatment in cerebral ischemia. Some studies suggest that hyperthermia may promote clot lysis. We hypothesized that low body temperature would prolong time to spontaneous clot lysis resulting in an association between low body temperature and severe neurological deficits in the early phase of ischemic stroke.

Methods: In this prospective study, patients $(n=516)$ exhibiting ischemic stroke with symptom onset within 6 hours were included. Body temperature and National Institute of Health Stroke Scale (NIHSS) score were registered on admission. Because low body temperature on admission may be secondary to immobilization due to large stroke, separate analyses were performed on patients with cerebral hemorrhage admitted within 6 hours $(n=85)$.

Results: Linear regression showed that low body temperature on admission was independently associated with a high NIHSS score within 6 hours of stroke onset in patients with ischemic stroke $(P<0.001)$. The association persisted when NIHSS was measured at 24 hours after admission. No such associations were found in patients with cerebral hemorrhage admitted within 6 hours of stroke onset.

Conclusion: Our study suggests that low body temperature within 6 hours of symptom onset is associated with severe ischemic stroke. This is in support of our hypothesis, although other contributing mechanisms cannot be excluded.

Keywords: body temperature, cerebral infarction, cerebral hemorrhage, clot lysis

\section{Introduction}

Hypothermia is generally known as a potent neuroprotectant in cerebral ischemia. Animal studies have shown an association between increased body temperature and increased stroke volume. ${ }^{1,2}$ Observation studies in stroke patients have shown an association between increased body temperature within the first 12 to 24 hours of stroke onset, and poor prognosis. ${ }^{3-5}$ This was confirmed in a large meta-analysis including patients with cerebral infarction, cerebral hemorrhage, and neurological injury. ${ }^{6}$

Some studies also suggest that temperature may affect clot lysis. In this context, it has been reported that streptokinase increases fibrinolysis at higher temperatures, and an in vitro study showed less clot lysis at lower temperatures when tissue plasminogen activator (tPA) was added to clot suspensions. ${ }^{7,8}$ Heating the tPA resulted in faster clot lysis in patients with acute lower limb ischemia when treated with catheter-directed thrombolysis. ${ }^{9}$
Correspondence: Christopher E Kvistad Department of Neurology, Haukeland University Hospital, University of Bergen, N-502I Bergen, Norway

Tel +47559750 40

Fax +47 559 75I 64

Email echr@helse-bergen.no 
We recently reported that a beneficial effect of high body temperature on clot lysis may be more important than the neuroprotective effect of low body temperature in the early phase after cerebral infarction in patients treated with tPA. ${ }^{10}$ This may suggest caution is required when lowering body temperature in stroke patients receiving IPA in the early phase.

In the present study we aimed to further analyze the effect of body temperature on the severity of stroke symptoms in the early phase after onset of ischemic stroke. Many patients experience rapid spontaneous clot lysis thereby improving prognosis. We hypothesized that low body temperature prolongs time to spontaneous clot lysis, suggesting that severe neurological deficits are associated with low body temperature in the early phase of ischemic stroke.

\section{Methods}

All consecutive patients with acute cerebral infarction (the index stroke) admitted to the Stroke Unit, Department of Neurology, Haukeland University Hospital between February 2006 and February 2010 were prospectively registered in a database (The Bergen NORSTROKE Registry). The stroke center at Haukeland University hospital (catchment area approximately 250,000 inhabitants) received all patients admitted with a possible stroke, regardless of age or stroke severity. In addition, patients with stroke aged $<60$ years from a neighboring area (approximately 120,000 inhabitants) were admitted. Patients may have been admitted directly without prior medical examination if they presented with persistent or transient acute symptoms that included sudden loss of strength in one body half, sudden loss of speech, and/or sudden loss of vision in one eye. If symptoms started within the last 6 hours, the patient was brought in by an emergency ambulance. Cerebral infarction was defined in accordance with the Baltimore-Washington Cooperative Young Stroke Study criteria comprising neurological deficits lasting more than 24 hours because of ischemic lesions or transient ischemic attacks where computed tomography (CT) or magnetic resonance imaging (MRI) showed infarctions related to the clinical findings. ${ }^{11}$

Baseline body temperature was measured upon admission to the hospital. Prior to temperature measurement, no temperature intervention (paracetamol or intravenous administration of fluids) was provided. During the period of February 2006 to September 2009, patients' temperature was measured primarily with an infrared tympanic temperature device (LighTouchLTX; Exergen Corp, Watertown, MA). The precision of this method has been validated in several studies. ${ }^{12-15}$ Between September 2009 and February 2010, patients' temperature was primarily measured using a temporal artery thermometer (Exergen TemporalScanner; Exergen Corp). This thermometer has a reported accuracy of $\pm 0.1^{\circ} \mathrm{C} .{ }^{16} \mathrm{In}$ a study of 57 critically ill patients, no significant difference was found between the pulmonary artery and temporal artery measurements. ${ }^{17}$

We performed a separate Student's $t$-test analysis comparing the body temperatures of the patients admitted before September 2009 with the body temperatures of the patients admitted after September 2009, and found no significant difference. The National Institute of Health Stroke Scale (NIHSS) was used to assess stroke severity on admission. tPA was administered to eligible patients according to the SITS protocol. In addition, tPA was administered in the 3 to 4.5 hour timeframe and to patients $>80$ years of age based on a case by case evaluation. Short-term outcomes were defined by the modified Rankin Scale (mRS) score on day 7 or at discharge, if discharged earlier.

Current smoking was defined as smoking at least one cigarette per day. Diabetes mellitus was considered present if the patient was on a glucose-lowering diet or medication. Renal impairment was defined as a serum creatinine level above $100 \mu \mathrm{mol} / \mathrm{L}$ on admission. ${ }^{18}$

Hypertension was considered present if diagnosed by a physician any time before stroke onset. Atrial fibrillation required electrocardiography (ECG) confirmation any time prior to stroke onset or during the hospital stay. Etiology was determined by the Trial of Org 10172 in Acute Stroke Treatment classification (TOAST) and classified as large artery atherosclerosis, cardioembolism, small vessel disease, other, and unknown. ${ }^{19}$

\section{Statistics}

The study population included patients with ischemic stroke admitted within 6 hours of symptom onset. Student's $t$-test was used for continuous variables. Fisher's exact test and chi-square test were used to analyze categorical variables. A linear regression analysis was performed with NIHSS score on admission as the dependent variable. NIHSS score was treated as a continuous variable. A logistic regression analysis was performed with mRS $0-2$ or 3-6 as the dependent variable, while sex, age, and temperature on admission were the independent variables. The relation between body temperature and NIHSS score on admission was visualized by Lowess function. Because low body temperature on admission may be secondary to immobilization due to large stroke, a separate analysis was performed on patients with cerebral hemorrhage admitted within 6 hours. STATA 11 (StataCorp, College Station, TX) was used for analyses. 


\section{Results}

In total, 516 patients with ischemic stroke were admitted within 6 hours of stroke onset, with a total of $212(41.1 \%)$ females and 304 (58.9\%) males. Table 1 shows the characteristics of these patients. Mean age was 70.4 years. On admission, median NIHSS score was 4 (interquartile range 2-11), and mean body temperature was $36.6^{\circ} \mathrm{C}$. Median time between ictus and temperature measurement was 109.7 minutes (interquartile range, 73.1-188.4). A total of 85 patients admitted within 6 hours of stroke onset had cerebral hemorrhage; $56(66 \%)$ males and 29 (34\%) females. Mean age was 71.5 years and median NIHSS score on admission was 13 (interquartile range, 6-21). An analysis of differences in baseline characteristics (the same variables were used as in Table 1) of patients with high and low body temperature on admission (cutoff value median $36.6^{\circ} \mathrm{C}$ ) was performed. In the ischemic group there were more males in the group with low temperatures $(P=0.009)$. There was also a significantly longer duration between symptom onset and temperature measurement in the patients with high temperatures $(P<0.001)$. There were no significant baseline differences in the hemorrhage group.

Table I Demography of patients with acute cerebral infarction admitted within 6 hours $(n=516)$

\begin{tabular}{|c|c|}
\hline Age, mean (SD) & $70.4(14.3)$ \\
\hline Female & $212(4 \mid .1 \%)$ \\
\hline Male & $304(58.9 \%)$ \\
\hline NIHSS score on admission, median (IQR) & $4(2-11)$ \\
\hline Body temperature on admission, mean (SD) & $36.6(0.6)$ \\
\hline $\begin{array}{l}\text { Minutes between ictus and temperature } \\
\text { measurement, median (IQR) }\end{array}$ & $109.7(73.1-188.4)$ \\
\hline Systolic blood pressure on admission, mean (SD) & $165(29)$ \\
\hline Diastolic blood pressure on admission, mean (SD) & $86(18)$ \\
\hline Glucose on admission, mean (SD) & $6.9(2.5)$ \\
\hline mRS score day 7, median (IQR) & $2(I-4)$ \\
\hline Hypertension & $253(49.4 \%)$ \\
\hline Diabetes mellitus & $65(12.8 \%)$ \\
\hline Renal impairment & $102(19.7 \%)$ \\
\hline Atrial fibrillation & I5I (26.3\%) \\
\hline Prior cerebral infarction & $70(13.7 \%)$ \\
\hline Myocardial infarction & $86(16.1 \%)$ \\
\hline Statin medication & $126(24.4 \%)$ \\
\hline Vitamin $\mathrm{K}$ antagonist medication & $44(8.5 \%)$ \\
\hline \multicolumn{2}{|l|}{ TOAST } \\
\hline Atherosclerosis & $63(12.2 \%)$ \\
\hline Cardiac embolism & 175 (33.9\%) \\
\hline Small vessel disease & $40(7.8 \%)$ \\
\hline Other & II (2.I\%) \\
\hline Unknown & $227(44.0 \%)$ \\
\hline
\end{tabular}

Abbreviations: IQR, interquartile range; NIHSS, National Institutes of Health Stroke Scale; SD, standard deviation; TOAST, Trial of Org 10172 in Acute Stroke Treatment.
Figure 1 shows the relationship between body temperature on admission within 6 hours of stroke onset in patients with ischemic stroke and their corresponding NIHSS scores. The correlation between NIHSS score and body temperature on admission was $\mathrm{r}=-0.17(P<0.001)$. There was no correlation between NIHSS score and body temperature on admission within 6 hours of stroke onset in patients with cerebral hemorrhage $(P=0.58)$.

Linear regression with NIHSS score on admission as the dependent variable showed that low body temperature on admission was independently associated with high NIHSS score within 6 hours of stroke onset in patients with ischemic stroke (Table 2). No such association was found in patients with cerebral hemorrhage admitted $<6$ hours (Table 2 ). Linear regression still showed a significant association between low body temperature measured on admission and high NIHSS score measured 24 hours after admission in ischemic stroke (Table 3). Modified Rankin Scale was assessed at day 7 after admission or at discharge, if discharged earlier. When dichotomized $-\mathrm{mRS}$ 0-2 as favorable outcome and mRS 3-6 as unfavorable outcome - we found a significant association between low body temperature and unfavorable outcome in ischemic stroke on logistic regression analysis with $\mathrm{mRS}$ 0-2 or 3-6 as the dependent variable, and gender, age, and temperature on admittance as the independent variables (odds ratio $=0.69, P=0.008$ ) (analysis not shown).

\section{Discussion}

Our findings support our hypothesis that in patients with ischemic stroke, low body temperature was associated with severe neurological deficits on admission within 6 hours of stroke onset. Two possible pathophysiological mechanisms may explain this result. Low body temperature may cause larger clot formation than high body temperature due to a larger imbalance between coagulation and lysis in favor of coagulation at low body temperature. This mechanism is speculative since other studies have shown that even mild hypothermia has been demonstrated to significantly impair coagulation resulting in prolonged coagulation time. ${ }^{20,21}$ Another possible mechanism is that spontaneous lysis takes place more slowly at low body temperature, resulting in prolonged time to spontaneous recanalization, which may be related to more severe neurological deficits in the early phase of ischemic stroke. Also this hypothesis remains largely speculative given that we do not have data or imaging regarding vessel occlusion and recanalization.

Our results may explain the disappointing results of previous attempts to treat stroke patients with different 


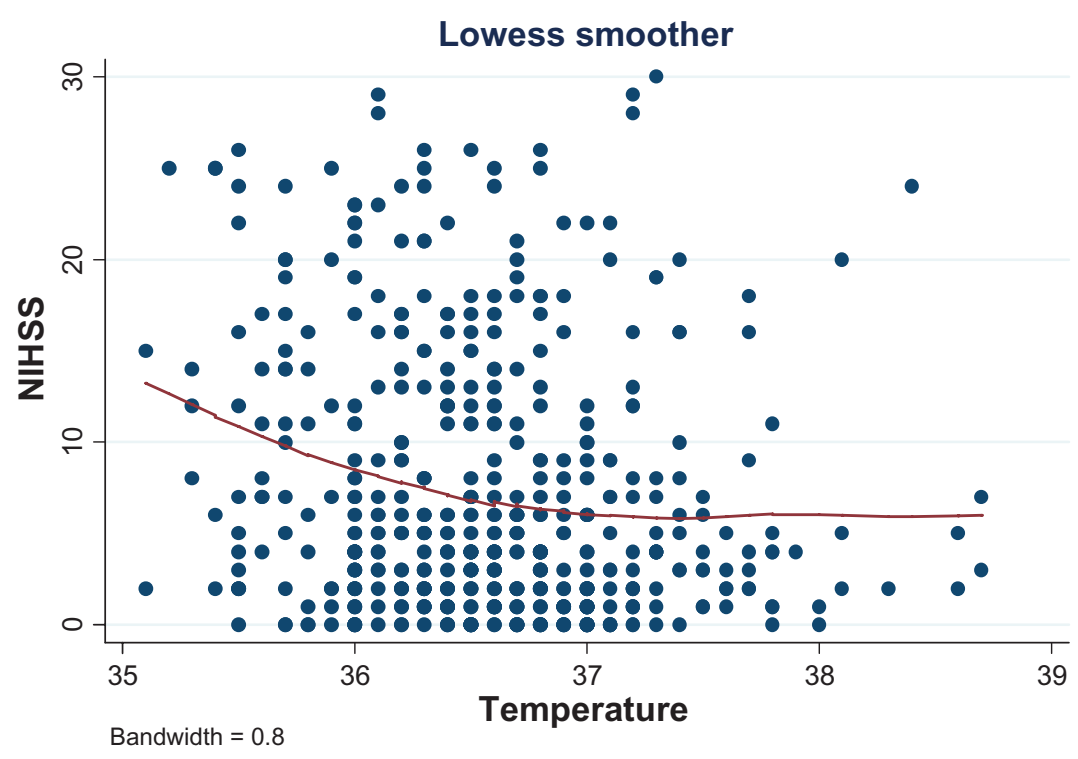

Figure I The relationship between NIHSS score and body temperature on admission in patients with ischemic stroke. Abbreviation: NIHSS, National Institutes of Health Stroke Scale.

cooling strategies. ${ }^{22-24}$ A systematic review of clinical randomized trials could not provide evidence that either pharmacological or physical temperature-lowering in the acute phase of stroke decreases the risk of death or dependence on assistance with daily function. ${ }^{25}$ Although cooling provides a neuroprotective effect, the prolonged time to spontaneous recanalization may negate this benefit. An animal study conducted to assess the safety of sonothrombolysis showed that insonation causes a moderate increase in brain temperature $\left(0.9^{\circ} \mathrm{C}\right)$, but without histopathological changes. ${ }^{26}$ It is possible that targeted warming of the blood clot with sonothrombolysis and simultaneous cooling of the brain is a better strategy in

Table 2 Linear regression analyses with NIHSS score on admission as dependent variable

\begin{tabular}{|c|c|c|c|c|}
\hline & \multicolumn{2}{|c|}{$\begin{array}{l}\text { Cerebral } \\
\text { infarction } \\
<6 \text { hours }\end{array}$} & \multicolumn{2}{|c|}{$\begin{array}{l}\text { Cerebral } \\
\text { hemorrhage } \\
<6 \text { hours }\end{array}$} \\
\hline & Beta & $P$ & Beta & $P$ \\
\hline Sex & 0.03 & 0.49 & 0.18 & 0.13 \\
\hline Age & 0.15 & 0.001 & -0.02 & 0.87 \\
\hline Body temperature & -0.17 & $<0.001$ & 0.10 & 0.41 \\
\hline Systolic blood pressure & -0.12 & 0.006 & -0.06 & 0.60 \\
\hline Glucose & 0.08 & 0.06 & 0.30 & 0.01 \\
\hline Atrial fibrillation & 0.10 & 0.02 & 0.001 & 0.95 \\
\hline Prior cerebral infarction & -0.09 & 0.04 & 0.05 & 0.66 \\
\hline $\begin{array}{l}\text { Time ictus-temperature } \\
\text { measurement }\end{array}$ & -0.18 & $<0.00 \mathrm{I}$ & -0.15 & 0.18 \\
\hline
\end{tabular}

Abbreviation: NIHSS, National Institutes of Health Stroke Scale. patients with ischemic stroke in the early phase of stroke onset.

Compatible with this study, a prospective study of 725 patients with stroke admitted within 6 hours of symptom onset found a relationship between low body temperature on admission and stroke severity. ${ }^{5}$ It was assumed that the severe stroke patients would lose body temperature faster during transportation because of lack of muscle activity resulting in a lower body temperature on admission. Likewise, a recent study examining 1332 stroke patients admitted within 12 hours of symptom onset showed that patients with severe stroke had relatively low body temperatures at baseline (median time from stroke onset to measurement of body

Table 3 Linear regression analyses with NIHSS score 24 hours after admission as dependent variable (independent variables on admission)

\begin{tabular}{|c|c|c|c|c|}
\hline & \multicolumn{2}{|c|}{$\begin{array}{l}\text { Cerebral } \\
\text { infarction } \\
<6 \text { hours }\end{array}$} & \multicolumn{2}{|c|}{$\begin{array}{l}\text { Cerebral } \\
\text { hemorrhage } \\
<6 \text { hours }\end{array}$} \\
\hline & Beta & $P$ & Beta & $P$ \\
\hline Sex & 0.04 & 0.50 & 0.10 & 0.62 \\
\hline Age & 0.19 & 0.001 & -0.07 & 0.75 \\
\hline Body temperature & -0.20 & $<0.001$ & 0.07 & 0.72 \\
\hline Systolic blood pressure & -0.16 & 0.003 & -0.13 & 0.51 \\
\hline Glucose & 0.07 & 0.20 & -0.09 & 0.63 \\
\hline Atrial fibrillation & 0.04 & 0.54 & -0.28 & 0.16 \\
\hline Prior cerebral infarction & -0.07 & 0.22 & -0.35 & 0.006 \\
\hline $\begin{array}{l}\text { Time ictus-temperature } \\
\text { measurement }\end{array}$ & -16 & 0.003 & 0.01 & 0.97 \\
\hline
\end{tabular}

Abbreviation: NIHSS, National Institutes of Health Stroke Scale. 
temperature was 360 minutes), conceivably because of a faster loss of body temperature before hospital admission as a result of less muscle activity. ${ }^{27}$

Because immobilization after severe stroke may possibly result in low body temperature, we analyzed the relationship between body temperature and severity of neurological deficits in patients admitted with cerebral hemorrhage within 6 hours of symptom onset. We did not find an association between neurological deficits and body temperature in these patients. It seems unlikely that there is an association between body temperature and neurological deficits in patients with ischemic stroke due to immobilization, but no such association in patients with cerebral hemorrhage.

There is a discrepancy between our results and the results found in other studies which have mainly found an association between high body temperature and severe stroke..$^{3-5,28,29}$ This discrepancy may be due to differences in study design. We were interested in the possible effect of body temperature on clot lysis, which may be best investigated in the very early phase after symptom onset. Most studies have evaluated the association between body temperature and severity of stroke among patients admitted more than 6 hours after stroke onset. Our findings suggest that the beneficial effect of low body temperature on neural tissue may outweigh the beneficial effect of high body temperature on clot lysis, but only beyond the early phase (ie, more than a few hours after stroke onset). Future studies should stratify patients according to the different timeframes of the different pathophysiological mechanisms in acute stroke. In addition, many studies have included both patients with cerebral infarction and cerebral hemorrhage. Given that temperature may affect these patient groups differentially, it is important to study each group separately.

A strength of this study is that the study protocol employed ensured identical monitoring of the patients in a single healthcare center. However, it should be noted that there are some limitations in the present study. First, systematic transcranial Doppler examinations were not performed. We therefore have no data to correlate body temperature with recanalization. Although the correlation between NIHSS score and body temperature was significant, the correlation was not particularly robust. We acknowledge that our results can possibly be explained by immobility and subsequent increased temperature loss in patients with larger strokes, although we could not find this association in patients with cerebral hemorrhage. The lack of such an association in patients with cerebral hemorrhage could, however, be explained by the small number of patients in this group. Nevertheless, including only the first 80 patients with cerebral infarction admitted within 6 hours still illustrated a highly significant association between stroke severity and body temperature (analysis not shown).

In conclusion, our study suggests that low body temperature within 6 hours of symptom onset is associated with more severe stroke. This may be due to a detrimental effect of low body temperature on clot lysis, although other contributing mechanisms cannot be excluded.

\section{Disclosure}

This study was not funded by any external source. There was no conflict of interest.

\section{References}

1. Dietrich WD, Halley M, Valdes I, Busto R. Interrelationships between increased vascular permeability and acute neuronal damage following temperature-controlled brain ischemia in rats. Acta Neuropathol. 1991;81(6):615-625.

2. Huang ZG, Xue D, Preston E, Karbalai H, Buchan AM. Biphasic opening of the blood-brain barrier following transient focal ischemia: effects of hypothermia. Can J Neurol Sci. 1999;26(4):298-304.

3. Jorgensen HS, Reith J, Pedersen PM, Nakayama H, Olsen TS. Body temperature and outcome in stroke patients. Lancet. 1996; 348(9021):193.

4. Castillo J, Davalos A, Marrugat J, Noya M. Timing for fever-related brain damage in acute ischemic stroke. Stroke. 1998;29(12):2455-2460.

5. Boysen G, Christensen H. Stroke severity determines body temperature in acute stroke. Stroke. 2001;32(2):413-417.

6. Greer DM, Funk SE, Reaven NL, Ouzounelli M, Uman GC. Impact of fever on outcome in patients with stroke and neurologic injury: a comprehensive meta-analysis. Stroke. 2008;39(11):3029-3035.

7. Mumme A, Kemen M, Homann HH, Zumtobel V. The temperature dependence of fibrinolysis with streptokinase. Dtsch Med Wochenschr. 1993;118(44):1594-1596.

8. Yenari MA, Palmer JT, Bracci PM, Steinberg GK. Thrombolysis with tissue plasminogen activator (tPA) is temperature dependent. Thromb Res. 1995;77(5):475-481.

9. Tsetis DK, Katsamouris AN, Giannoukas AD, et al. Potential benefits from heating the high-dose rtPA boluses used in catheter-directed thrombolysis for acute/subacute lower limb ischemia. JEndovasc Ther. 2003;10(4):739-744.

10. Naess H, Idicula T, Lagallo N, Brogger J, Waje-Andreassen U, Thomassen L. Inverse relationship of baseline body temperature and outcome between ischemic stroke patients treated and not treated with thrombolysis: the Bergen stroke study. Acta Neurol Scand. 2010;122(6):414-417.

11. Johnson CJ, Kittner SJ, McCarter RJ, et al. Interrater reliability of an etiologic classification of ischemic stroke. Stroke. 1995;26(1):46-51.

12. Summers S. Axillary, tympanic, and esophageal temperature measurement: descriptive comparisons in postanesthesia patients. J Post Anesth Nurs. 1991;6(6):420-425.

13. Erickson RS, Kirklin SK. Comparison of ear-based, bladder, oral, and axillary methods for core temperature measurement. Crit Care Med. 1993;21(10):1528-1534.

14. Klein DG, Mitchell C, Petrinec A, et al. A comparison of pulmonary artery, rectal, and tympanic membrane temperature measurement in the ICU. Heart Lung. 1993;22(5):435-441.

15. Stewart JV, Webster D. Re-evaluation of the tympanic thermometer in the emergency department. Ann Emerg Med. 1992;21(2): $158-161$. 
16. Lee G, Flannery-Bergey D, Randall-Rollins K, et al. Accuracy of temporal artery thermometry in neonatal intensive care infants. $A d v$ Neonatal Care. 2011;11(1):62-70.

17. Myny D, De Waele J, Defloor T, Blot S, Colardyn F. Temporal scanner thermometry: a new method of core temperature estimation in ICU patients. Scott Med J. 2005;50(1):15-18.

18. Rustad P, Felding P, Franzson L, et al. The Nordic Reference Interval Project 2000: recommended reference intervals for 25 common biochemical properties. Scand J Clin Lab Invest. 2004;64(4):271-284.

19. Adams HP Jr, Bendixen BH, Kappelle LJ, et al. Classification of subtype of acute ischemic stroke. Definitions for use in a multicenter clinical trial. TOAST. Trial of Org 10172 in Acute Stroke Treatment. Stroke. 1993;24(1):35-41.

20. Rajagopalan S, Mascha E, Na J, Sessler DI. The effects of mild perioperative hypothermia on blood loss and transfusion requirement. Anesthesiology. 2008;108(1):71-77.

21. Douning LK, Ramsay MA, Swygert TH, et al. Temperature corrected thrombelastography in hypothermic patients. Anesth Analg. 1995;81(3):608-611.

22. den Hertog HM, van der Worp HB, van Gemert HM, et al. The Paracetamol (Acetaminophen) In Stroke (PAIS) trial: a multicentre, randomised, placebo-controlled, phase III trial. Lancet Neurol. $2009 ; 8(5): 434-440$
23. De Georgia MA, Krieger DW, Abou-Chebl A, et al. Cooling for Acute Ischemic Brain Damage (COOL AID): a feasibility trial of endovascular cooling. Neurology. 2004;63(2):312-317.

24. Hemmen TM, Raman R, Guluma KZ, et al. Intravenous thrombolysis plus hypothermia for acute treatment of ischemic stroke (ICTuS-L): final results. Stroke. 2010;41(10):2265-2270.

25. Den Hertog HM, van der Worp HB, Tseng MC, Dippel DW. Cooling therapy for acute stroke. Cochrane Database Syst Rev. 2009;1:CD001247.

26. Fatar M, Stroick M, Griebe M, Alonso A, Hennerici MG, Daffertshofer M. Brain temperature during $340-\mathrm{kHz}$ pulsed ultrasound insonation: a safety study for sonothrombolysis. Stroke. 2006;37(7):1883-1887.

27. den Hertog HM, van der Worp HB, van Gemert HM, et al. An early rise in body temperature is related to unfavorable outcome after stroke: data from the PAIS study. J Neurol. 2011;258(2):302-307.

28. Hindfelt B. The prognostic significance of subfebrility and fever in ischaemic cerebral infarction. Acta Neurol Scand. Jan 1976;53(1): 72-79.

29. Castillo J, Martinez F, Leira R, Prieto JMM, Lema M, Noya M. Mortality and morbidity of acute cerebral infarction related to temperature and basal analytic parameters. Cerebrovasc Dis. 1994;4:56-71.
Vascular Health and Risk Management

\section{Publish your work in this journal}

Vascular Health and Risk Management is an international, peerreviewed journal of therapeutics and risk management, focusing on concise rapid reporting of clinical studies on the processes involved in the maintenance of vascular health; the monitoring, prevention and treatment of vascular disease and its sequelae; and the involvement of

\section{Dovepress}

metabolic disorders, particularly diabetes. This journal is indexed on PubMed Central and MedLine. The manuscript management system is completely online and includes a very quick and fair peer-review system, which is all easy to use. Visit http://www.dovepress.com/ testimonials.php to read real quotes from published authors. 